\title{
The design and development of automatic spraying system for automotive wheel hub based on PLC control
}

\author{
Chang Zhong Wu ${ }^{\mathrm{a}}$, Xiu Guo Wei ${ }^{\mathrm{b}}$ and Qing Chic \\ ${ }^{1}$ School of Mechanical Engineering, University of Jinan, Jinan, China \\ ${ }^{2}$ School of Mechanical Engineering, University of Jinan, Jinan, China \\ ${ }^{3}$ School of Mechanical Engineering, University of Jinan, Jinan, China

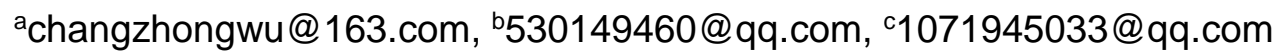

\begin{abstract}
Keywords: Wheel hub, Automatic spraying, Electrostatic spray gun,PLC
Abstract. In the modern auto parts manufacturing, the production of hub is a very important branch. In order to improve the beauty of the wheel hub and corrosion resistance, the finished wheel hub needs to be sprayed. In this paper, the automatic spraying system of the hub is designed by applying the existing automatic electrostatic spraying gun, and the automatic spraying system of the car hub is designed based on PLC control. The whole system is based on the Siemens s7-200 (cpu244) controller. The system can replace the traditional manual spraying, which saves paint, improves efficiency and quality, and ensures employee's health, simultaneously.
\end{abstract}

\section{Introduction}

Spraying is the process of coating the workpiece surface evenly with spray gun. In manufacturing, spraying is a very important process, and it observably determines the corrosion, rust and beauty of the workpiece. Spraying technology is widely used in military, aerospace, automobile, hardware and other fields. Electrostatic spraying is a method of spraying the coating particles on the surface of the workpiece by using high voltage electrostatic field to direct the negatively charged coating particles in the opposite direction of the electric field. Spraying can make the surface of the workpiece evenly coated and not drop easily. At the same time, the technology is stable and reliable, with low cost and high efficiency.

\section{The description of the wheel hub automatic spraying system}

Automatic spraying system can be divided into mechanical part, software control part and electronic detection part, respectively. The mechanical part adopts the chain transmission mode, which is simple, reliable, easy to control and low cost. The software control part mainly relies on microcontroller such as microcontroller, PLC, etc. The electronic detection is mainly used to detect the various data of the workpiece, such as the position of the workpiece, the speed, the size and so on.

The design requirement. The main design features of the design of wheel automatic spraying system mentioned in the present paper are wheel hub transit speed, position, size detection, under the condition of the production line of continuous work non-stop to complete different batch, and different specification wheel hub of the spraying operations.

Overall scheme design. To achieve automatic spraying, three spray guns are selected. Lateral surface spray coating and coating on the surface applies a spray gun, respectively. Spraying on the surface of the two spray gun is tilted, and the oblique Angle is $70^{\circ}$, which is different from traditional spraying. A center hole of a vertical hub, and one side of the center hole moves outward along the radius. Three spray guns work simultaneously, spraying the sides and upper surfaces respectively. The three spray guns are in the initial position before spraying. When the system detects that the hub is about to enter the spray area, the spray action will be determined according to the size, speed and position of the wheel hub detected. When the hub enters the spray area, the hub will rotate, and the 
three spray guns will move forward with the wheel hub. After spraying, three spray guns will return to the initial position and start the next round of spraying. Simplified spray action is shown in figure 2.1.

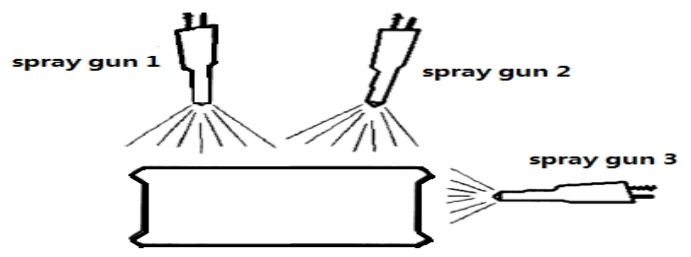

Figure 2.1 simplified spray action

Overall scheme design of mechanical system. The mechanical structure of the automatic spraying system consists of two degrees of freedom of reciprocating motion, namely the horizontal reciprocating motion of three spray guns. One degree of freedom along horizontal direction is used to drive the two spray guns that are sprayed on the surface, and the other degree of freedom is used to drive a spray gun that is sprayed on the side. Specifically, mechanical structure includes: ground chain transfer mechanism, supporting guide rail, rotation device, rack part, wheel hub tray, and spray gun mounting bracket.

Transmission scheme design. The transmission of the automatic spray system designed in this paper contains two chains: one is the chain drive of the hub conveyor and the other is the chain drive used for the rotation of wheel hub tray. In the chain of wheel hub lanes, USES is precision roller chain structure, which is in the hollow shaft pin chain in vertical shaft pin. Meanwhile, wheel hub tray can be fixed on the vertical axis pin, through the chain of transmission can drive the tray transport hub. It can drive the rotation of the chain wheel by controlling the motor. As shown in figure 3.1.

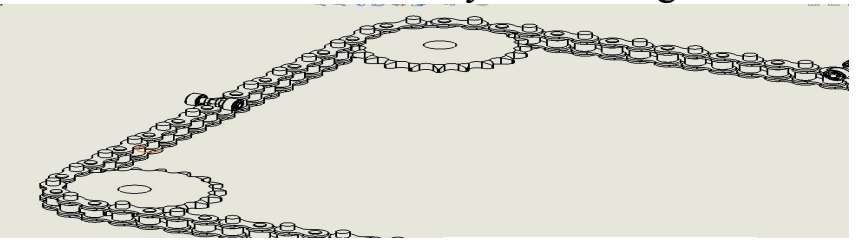

Figure 3.1 Chain drive

In the rotation drive of hub tray, it is also used to transfer torque with the meshing of sprocket and chain. When the hub pallet mechanism enters the spray area, the chain wheel on the hub tray is meshed with the chain on the rotation device, thus causing the rotation of the hub tray mechanism. As shown in figure 3.2 .

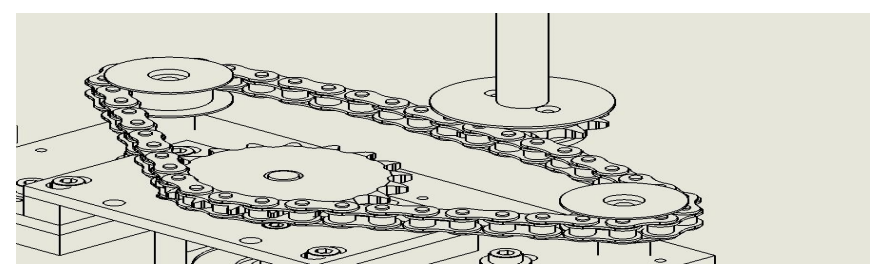

Figure 3.2 The chain drive of the rotation device

The design of the rotation device and hub tray. In automatic spraying system work time, as the hub is centrosymmetric artifacts, it needs only one gun to the side which can guarantee paint job completely. Therefore, we need to design the rotation device to achieve the purpose of the wheel rotation on entering the spraying area. In the spraying area, in order to guarantee the wheel hub transit uniform, spraying operations are not execute $d$ at this time. So when the wheel rotates, it need not work into the spraying area. To the side of the wheel hub it can be uniform coating, where wheel hub must be rotational. Rotation device includes an active chain wheel, motor, two driven wheels and the chain. By means of the sprocket wheel trays with rotation device on the gears mesh chain, achieve the goal of rotation. The working principle of the rotation device is shown in figure 3.3. 


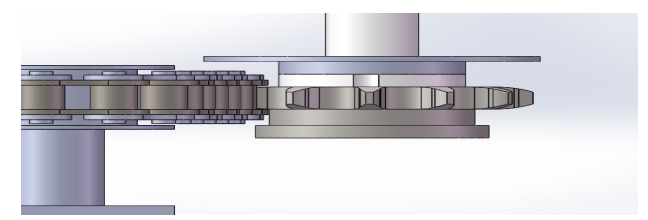

Figure 3.3 The chain drive of the rotation device

Wheel hub tray mainly consists of wheel hub support plate, chain wheel, bearing, etc. In actual production, the wheel hub is carried by the manipulator, and the center hole of the hub is positioned in the middle position of the support disk of the hub tray. The position of the hub tray contacts with the hub needs to be sprayed on the surface to prevent the abrasion of the metal surface of the center hole of the hub, affecting the assembly of the hub. The design of hub tray is shown in figure 3.4.

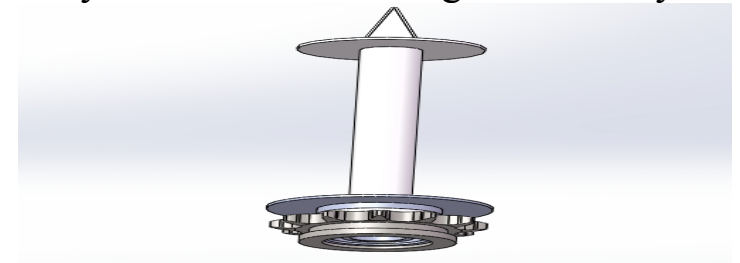

Figure 3.4 The chain drive of the rotation device

Frame structure design. In order to facilitate the positioning and assembly of the wheels and components of the manipulator, this paper designed the frame structure, which is shown in the figure 3.5. The structure of the frame is equipped with a sprocket, motor, guide rail, the installation position of the spraying room shell, and the support of the motor. The blowers are set at the bottom of the frame. The chain transmission device is arranged in the central area of the frame, which is about $750 \mathrm{~mm}$ in height, which is in line with the ergonomic design concept, which facilitates the installation and commissioning of other devices, and also facilitates the staff to check the production status of the observation line. The frame structure is shown in figure 3.5.

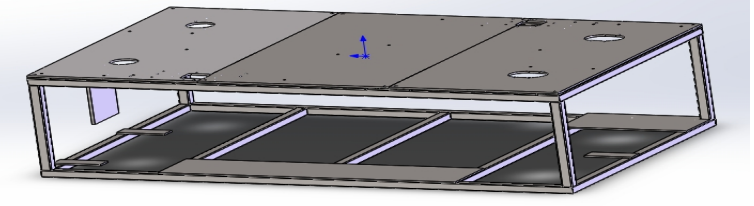

Figure 3.5 Frame structure

Design of spray gun mounting bracket. The fixed support is mounted on the mounting pedestal of the re-machine through the base, and the spray gun can be fixed on the machine. It is the same with the spray gun mounted on the surface and the side. The upper surface airbrush holder requires two mounting support rods, while the side nozzle holder only needs one. As shown in figure 3.6, the nozzle can be used to adjust the installation angle and direction of the gun through the nut and fastening screw after installation.

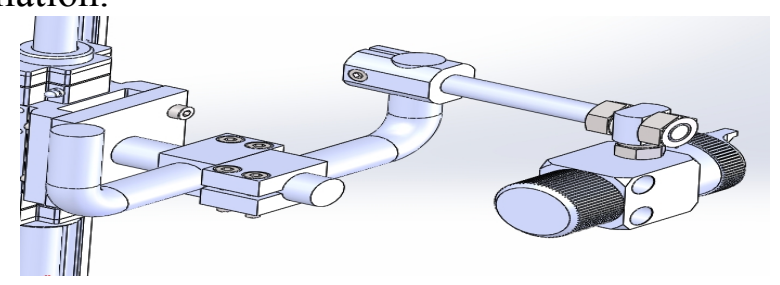

Figure 3.6 Spray-gun mounts

Control system master plan. At present, in industrial production, multi-purpose single chip, industrial control machine, and PLC are widely used as controller of control system. Considering the spraying operation conditions, dust, noise, etc, medium-sized equipment and spraying system, so we can choose high reliability, short development cycle, relatively low cost PLC as controller of automatic spraying systems. The movement of the gun and reset, is done by PLC control motor drive, in addition, we also design the power indicator light, fault indicator light and normal work lights, and start-stop buttons, abrupt stop button to display the working status and the operation of the control system and stop. The system scheme is shown in figure 4.1. 


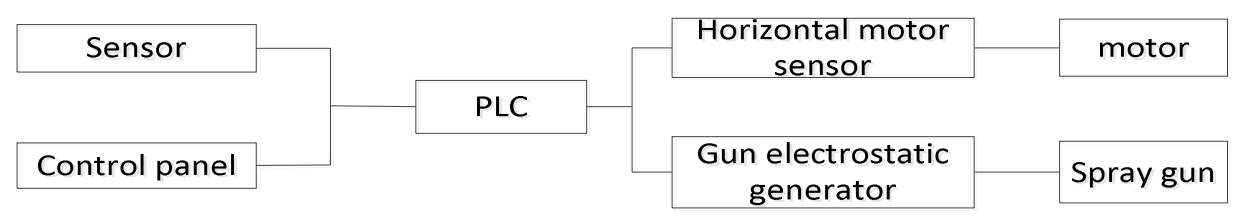

Figure 4.1 Control system scheme block diagram

The hardware system. The s7-200P used in this design is applicable to all walks of life, testing, monitoring and so on. The Siemens PLC s7-200 has excellent performances such as high reliability, rich set of instructions, easy to master and operate, rich built-in integration and real-time features, multiple extension modules. Siemens PLC s7-200 series has multiple models of CPU, and this paper chooses cpu224. The I/0 port assignment of PLC is shown in figure 4.2.

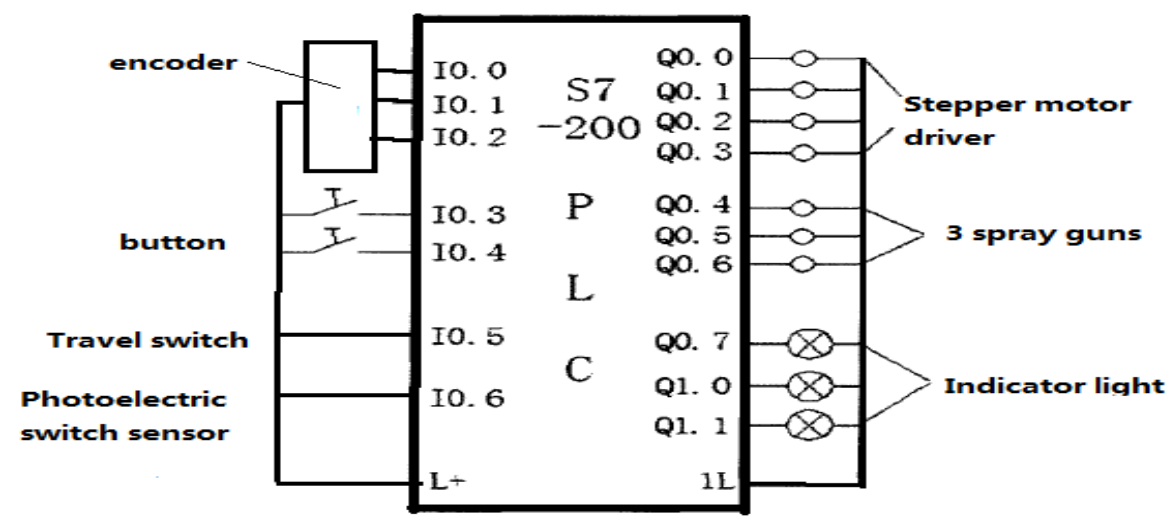

Figure 4.2 Port assignment diagram

Detection of speed of hub transport line. In order to ensure that the speed of the nozzle can be the same as the speed of the hub, the system is required to detect the speed of the hub transport line in real time. In this paper, an incremental rotary encoder is used to measure the wheel hub speed. The rotary encoder is a device for measuring speed, which is divided into single and double output. Single-channel output refering to the output of the rotary encoder is a set of pulses, and dual output refers to the output pulse phase difference of 90 degrees, the two groups in the two groups of pulse not only can measure the rotational speed, also can judge the direction of rotation. When used, the rotary encoder is connected to the drive motor of the hub conveyor line. Rotary encoder can convert the angular displacement of the motor under test to digital signals, so the output of the rotary encoder pulse signal can directly import PLC, by using the PLC high-speed counter to count the pulse signal, in order to obtain the measured results. The rotary encoder has 5 leads, 3 of which are pulse output lines and 2 are power lines. The source of the encoder can be an external power source, or directly used the DC24V power supply of PLC. The pulse output line of A, B and Z is directly connected with the input terminal of PLC. The rotating encoder wiring diagram is shown in figure 4.3.

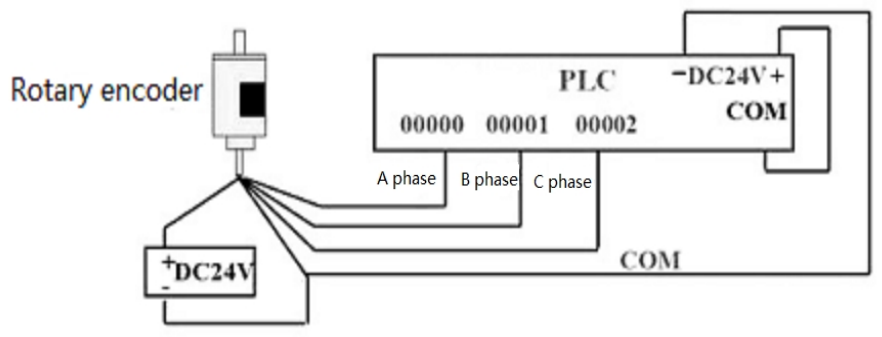

Figure 4.3 Rotate encoder wiring diagram

Reset to control the spray gun. The three spray guns controlled by the system are in the horizontal direction of reciprocating linear motion. After the wheel hub spraying, the spray gun is returned to the origin to carry out the next spraying action. The system selects the travel switch to control the nozzle reset. In the electrical control system, the function of the stroke switch is to realize 
sequence control, positioning control and location state, to control the travel and limit the protection of mechanical equipment. It consists of an operation head, a contact system and a shell. In spraying, the stroke switch is installed in a pre-arranged position, when installed on the spray gun in the moving parts of module impact travel switch, trip switch contact action, gun control movement control of the motor stopped, so as to achieve the goal of restoration.

\section{Conclusions}

This paper presents a design of automotive hub automatic spraying system based on PLC control, which includes mechanical structure and control system. For the transmission mode of the system, the chain transmission mode is adopted, and the chain drive is more suitable for the bad spraying environment with more dust than other transmission modes. For the system's driving mode, it adopts stepper motor as the driving original, in addition, the design calculation is carried out on the frame of the system, the nozzle fixation bracket, wheel hub tray and so on. For the control system, s7-200 (CPU244)PLC is used as the controller of the system. In the design process, the parameters of the whole system are selected in combination with the field investigation and actual production.

\section{References}

[1]FM453 Servo Drive/Step Drive Positioning Module. SIEMENS, 2012

[2]SIMODRIVE 611 universal Control Components for Closed-Loop Speed Control and Positioning. SIEMENS, 2015

[3]SIMATIC S7-400 Power PLC. SIEMENS, 2009

[4] R Jeffery. Integrating Scales Weight Information and PLC. Australian Journal of Instrumentation and Control. 1998(13): 10-16

[5]M Ogawa, Y Henmi. Recent Developments on PC+PLC based Control Systems for Beer Brewery Process Automation Applications [C], In Proceedings of SICE-ICASE international Joint Conference 2015: 1053-1056 